\title{
POSITIVE SOLUTIONS OF NONLOCAL SINGULAR BOUNDARY VALUE PROBLEMS
}

\author{
RAVI P. AGARWAL \\ Department of Mathematical Sciences, Florida Institute of Technology Melbourne, \\ Florida 32901-6975, USA \\ e-mail:agarwal@fit.edu \\ DONAL O'REGAN \\ Department of Mathematics, National University of Ireland, Galway, Ireland \\ e-mail:donal.oregan@nuigalway.ie \\ and SVATOSLAV STANĚK* \\ Department of Mathematical Analysis, Faculty of Science, Palacký University, \\ Tomkova 40, 77900 Olomouc, Czech Republic \\ e-mail:stanek@risc.upol.cz
}

(Received 3 October, 2003; accepted 12 May, 2004)

\begin{abstract}
The paper presents the existence result for positive solutions of the differential equation $(g(x))^{\prime \prime}=f\left(t, x,(g(x))^{\prime}\right)$ satisfying the nonlocal boundary conditions $x(0)=x(T), \min \{x(t): t \in J\}=0$. Here the positive function $f$ satisfies local Carathéodory conditions on $[0, T] \times(0, \infty) \times(\mathbb{R} \backslash\{0\})$ and $f$ may be singular at the value 0 of both its phase variables. Existence results are proved by Leray-Schauder degree theory and Vitali's convergence theorem.
\end{abstract}

2000 Mathematics Subject Classification. 34B16, 34B15.

1. Introduction. Let $T$ be a positive number, $J=[0, T]$ and $\mathbb{R}_{0}=\mathbb{R} \backslash\{0\}$. We shall discuss the singular differential equation

$$
(g(x(t)))^{\prime \prime}=f\left(t, x(t),\left(g(x(t))^{\prime}\right),\right.
$$

where $g \in C^{0}([0, \infty))$ and the positive function $f$ satisfies local Carathéodory conditions on $J \times(0, \infty) \times \mathbb{R}_{0}\left(f \in \operatorname{Car}\left(J \times(0, \infty) \times \mathbb{R}_{0}\right)\right)$ and $f$ may be singular at the value 0 of both its phase variables.

Furthermore we shall deal with the nonlocal boundary conditions

$$
x(0)=x(T), \quad \min \{x(t): t \in J\}=0 .
$$

We say that $x \in C^{0}(J)$ is a solution of the boundary value problem (BVP for short) (1.1), (1.2) if $g(x) \in A C^{1}(J)$ (functions having absolutely continuous derivative on $J$ ), $x$ satisfies the boundary conditions (1.2) and (1.1) holds a.e. on $J$.

In this paper we are interested in finding conditions on the functions $g$ and $f$ in (1.1) that guarantee the existence of positive solutions to BVP(1.1), (1.2). The existence

\footnotetext{
*Supported by grant no. 201/01/1451 of the Grant Agency of the Czech Republic and by the Council of the Czech Government J14/98:153100011.
} 
result is proved by regularization and sequential techniques. Any positive solution $x$ and $(g(x))^{\prime}$ for BVP (1.1), (1.2) 'go through' singularities of $f$ somewhere inside of $J$.

We show that our existence result for BVP (1.1), (1.2) can be applied to obtain solutions of BVP (1.3), (1.2), where

$$
\left(g(x(t)) x^{\prime}(t)\right)^{\prime}=f\left(t, x(t), g(x(t)) x^{\prime}(t)\right) .
$$

By a solution of $B V P(1.3),(1.2)$ we understand a function $x \in C^{1}(J)$ satisfying (1.2), $g(x) x^{\prime} \in A C(J)$ and (1.3) is true almost everywhere on $J$.

We note that only a few papers in the literature are devoted to the study of BVPs for differential equations of the form (1.1) (see [1], [7] and references therein). In [1] the authors consider via the method of lower and upper functions the Dirichlet problem with the differential equation $(P(x))^{\prime \prime}+f_{1}(t, x)=0$ where $P(z)=\int_{0}^{z} r(x) d x$, $r$ is a continuous function and $f_{1}$ satisfies local Carathéodory conditions. Existence results for solutions of $(P(x))^{\prime \prime}=q(t) f_{2}\left(t, x, x^{\prime}\right)$ with continuous $f_{2}$ satisfying the Dirichlet boundary conditions are given in [7]. Differential equations of the form $\left(g(x) x^{\prime}\right)^{\prime}=f_{3}\left(t, x, x^{\prime}\right)$ and two-point boundary conditions were considered (in the regular case also) in [7]. The Dirichlet problem for differential equations of the form $\left(r(x) x^{\prime}\right)^{\prime}=\mu q(t) f_{4}(t, x)$ where $f_{4}$ is singular at the value 0 of its phase variable $x$ was studied in [8]-[10]. In [2] the authors give conditions for the existence of positive solutions of a more general equation $\left(g(x)\left(x^{\prime}\right)^{\alpha}\right)^{\prime}=\mu q(t) f_{5}(t, x)\left(x^{\prime}\right)^{\beta}$ with $\alpha \in(0, \infty)$ and $\beta \in\{0,1\}$ satisfying the Dirichlet boundary conditions. Existence results for a functional differential equation with a nonlinear functional left hand side and nonlocal boundary conditions are presented in [4]. In all the papers above, BVPs are considered only for local boundary conditions and, in the case that differential equations are singular at their phase variables solutions 'start' and/or 'finish', at singular points (with the exception of [4] and [9]).

In this paper the following assumptions will be used.

$\left(H_{1}\right) g \in C^{0}([0, \infty))$ is increasing, $g(0)=0$ and $\lim _{u \rightarrow \infty} g(u)=\infty$.

$\left(H_{2}\right) g \in C^{0}([0, \infty))$ is positive and $\lim _{u \rightarrow \infty} G(u)=\infty$, where

$$
G(u)=\int_{0}^{u} g(s) d s, \quad u \in[0, \infty) .
$$

$\left(H_{3}\right) f \in \operatorname{Car}\left(J \times(0, \infty) \times \mathbb{R}_{0}\right)$ and there exists a positive constant $a \leq 1 / 2$ such that

$$
a \leq f(t, x, y) \text { for a.e. } t \in J \text { and each }(x, y) \in(0, \infty) \times \mathbb{R}_{0} .
$$

$\left(H_{4}\right)$ For a.e. $t \in J$ and each $(x, y) \in(0, \infty) \times \mathbb{R}_{0}$,

$$
f(t, x, y) \leq\left(h_{1}(x)+h_{2}(x)\right)\left(\omega_{1}(|y|)+\omega_{2}(|y|)\right),
$$

where $h_{1}, \omega_{1} \in C^{0}([0, \infty))$ are non-negative and non-decreasing, $h_{2}, \omega_{2} \in C^{0}((0, \infty))$ are positive and non-increasing.

$$
\begin{aligned}
\left(H_{5}\right) \int_{0}^{1} h_{2}\left(g^{-1}\left(s^{2}\right)\right) \omega_{2}(s) d s & <\infty \text { and } \\
& \lim _{u \rightarrow \infty} \int_{0}^{u} \frac{1}{K^{-1}\left(H_{1}(s)\right)} d s>\frac{T}{2},
\end{aligned}
$$


where

$$
\begin{gathered}
K(u)=\int_{0}^{u} \frac{s}{\omega_{1}(s+1)+\omega_{2}(s)} d s, \quad u \in[0, \infty), \\
H_{1}(u)=\int_{0}^{u}\left[h_{1}\left(g^{-1}(s)+1\right)+h_{2}\left(g^{-1}(s)\right)\right] d s, \quad u \in[0, \infty) . \\
\left(H_{6}\right) \int_{0}^{1} h_{2}\left(G^{-1}\left(s^{2}\right)\right) \omega_{2}(s) d s<\infty \text { and } \\
\lim _{u \rightarrow \infty} \int_{0}^{u} \frac{1}{K^{-1}\left(H_{2}(s)\right)} d s>\frac{T}{2},
\end{gathered}
$$

where

$$
H_{2}(u)=\int_{0}^{u}\left[h_{1}\left(G^{-1}(s)+1\right)+h_{2}\left(G^{-1}(s)\right)\right] d s, \quad u \in[0, \infty) .
$$

REMARK 1.1. Let assumptions $\left(H_{4}\right)$ and $\left(H_{5}\right)$ be satisfied. We show that the integral $\int_{0}^{u}\left(1 / K^{-1}\left(H_{1}(s)\right)\right) d s$ is convergent for all $u>0$. Since $h_{1}\left(g^{-1}(u)+1\right)+h_{2}\left(g^{-1}(u)\right) \geq$ $h_{2}\left(g^{-1}(1)\right)$ and $\omega_{1}(u+1)+\omega_{2}(u) \geq \omega_{2}(1)$ for $u \in[0,1]$, we have $H_{1}(u) \geq h_{2}\left(g^{-1}(1)\right) u$ and $K(u) \leq u^{2} /\left(2 \omega_{2}(1)\right)$ for these $u$. Hence $K^{-1}\left(H_{1}(u)\right) \geq \sqrt{2 h_{2}\left(g^{-1}(1)\right) \omega_{2}(1) u}$ for $u \in[0, \tau]$ with a $\tau>0$ and since $K^{-1}\left(H_{1}\right)$ is positive and continuous on $(0, \infty)$, we see that $\int_{0}^{u}\left(1 / K^{-1}\left(H_{1}(s)\right)\right) d s<\infty$ for all $u>0$. Analogously we can verify that $\int_{0}^{u}\left(1 / K^{-1}\left(H_{2}(s)\right)\right) d s<\infty$ for $u>0$ if assumptions $\left(H_{4}\right)$ and $\left(H_{6}\right)$ are satisfied.

The paper is organized as follows. In Section 2 we prove that the solvability of BVP (1.1), (1.2) is equivalent to that of BVP (2.1), (1.2) (Lemma 2.1). Section 3 deals with a sequence of auxiliary regular BVPs to BVP (2.1), (1.2) where the nonlinearities $f_{n}$ in the differential equations are regular functions on $J \times \mathbb{R}^{2}$. We give a priori bounds for their solutions $x_{n}$ (Lemma 3.3) and prove their existence (Lemma 3.4) using Leray-Schauder degree theory (see, for example, [5]). In addition, we show that the sequence $\left\{f_{n}\left(t, g^{-1}\left(x_{n}(t)\right), x_{n}^{\prime}(t)\right)\right\}$ is uniformly absolutely continuous on $J$ (Lemma 3.5). In Section 4 we present our main results: the existence of a positive solution to BVP (1.1), (1.2) (Theorem 4.1) and to BVP (1.3), (1.2) (Corollary 4.2). In limiting processes we use the Vitali's convergence theorem (see, for example, [3], [6]) since it is impossible to find a Lebesgue integrable majorant function for the sequence $\left\{f_{n}\left(t, g^{-1}\left(x_{n}(t)\right), x_{n}^{\prime}(t)\right)\right\}$ which is necessary for applying the Lebesgue dominated convergence theorem. We include also two examples (Examples 4.3 and 4.4) to illustrate our theory.

2. Lemma. Let assumptions $\left(H_{1}\right)$ and $\left(H_{3}\right)$ be satisfied. Together with the differential equation (1.1) we consider the differential equation

$$
x^{\prime \prime}(t)=f\left(t, g^{-1}(x(t)), x^{\prime}(t)\right) .
$$

We say that $x$ is a solution of equation (2.1) if $x \in A C^{1}(J)$ and $x$ satisfies (2.1) a.e. on $J$.

In the next lemma we give relations between solutions of BVP (1.1), (1.2) and BVP (2.1), (1.2). 
LEMMA 2.1. Let assumptions $\left(H_{1}\right)$ and $\left(H_{3}\right)$ be satisfied. If $x(t)$ is a solution of $B V P$ (1.1), (1.2), then the function $u(t)=g(x(t)), t \in J$, is a solution of $B V P(2.1),(1.2)$ and also conversely, if $x(t)$ is a solution of $B V P(2.1),(1.2)$, then the function $u(t)=g^{-1}(x(t))$, $t \in J$, is a solution of $B V P(1.1),(1.2)$.

Proof. Let $x$ be a solution of $\operatorname{BVP}(1.1),(1.2)$. Then $x \in C^{0}(J), g(x) \in A C^{1}(J)$ and $x$ satisfies (1.2). Set $u(t)=g(x(t))$ for $t \in J$. Then $u(0)=u(T), \min \{u(t): t \in J\}=0$, $u \in A C^{1}(J)$ and $u^{\prime \prime}(t)=(g(x(t)))^{\prime \prime}=f\left(t, x(t),\left(g(x(t))^{\prime}\right)=f\left(t, g^{-1}(u(t)), u^{\prime}(t)\right)\right.$ a.e. on $J$. Hence $u$ is a solution of BVP (2.1), (1.2).

Let $x$ be a solution of BVP (2.1), (1.2). Then $x$ satisfies (1.2) and $x \in A C^{1}(J)$. Let $u(t)=g^{-1}(x(t)), t \in J$. Then (1.2) holds with $u$ instead of $x, u \in C^{0}(J), g(u)=$ $x \in A C^{1}(J)$ and $(g(u(t)))^{\prime \prime}=x^{\prime \prime}(t)=f\left(t, g^{-1}(x(t)), x^{\prime}(t)\right)=f\left(t, u(t),\left(g(u(t))^{\prime}\right)\right.$ a.e. on $J$. Thus $u$ is a solution of BVP (1.1), (1.2).

REMARK 2.2. From Lemma 2.1 we see that solving BVP (1.1), (1.2) is equivalent to solving BVP (2.1), (1.2).

3. Auxiliary regular BVPs. For each $n \in \mathbb{N}$, define $f_{n} \in \operatorname{Car}\left(J \times \mathbb{R}^{2}\right)$ by

$$
f_{n}(t, x, y)= \begin{cases}f(t, x, y) \quad \text { for } t \in J, \quad x \geq \frac{1}{n},|y| \geq \frac{1}{n} \\ f\left(t, \frac{1}{n}, y\right) \quad \text { for } t \in J, x<\frac{1}{n},|y| \geq \frac{1}{n} \\ \frac{n}{2}\left[f_{n}\left(t, x, \frac{1}{n}\right)\left(y+\frac{1}{n}\right)-f_{n}\left(t, x,-\frac{1}{n}\right)\left(y-\frac{1}{n}\right)\right] \\ \quad \text { for } t \in J, x \in \mathbb{R}, y \in\left(-\frac{1}{n}, \frac{1}{n}\right) .\end{cases}
$$

Then $\left(H_{3}\right)$ and $\left(H_{4}\right)$ yield (for $n \in \mathbb{N}$ )

$$
a \leq f_{n}(t, x, y) \quad \text { for a.e. } t \in J \text { and each }(x, y) \in \mathbb{R}^{2}
$$

and

$$
f_{n}(t, x, y) \leq\left(h_{1}(x+1)+h_{2}(x)\right)\left(\omega_{1}(|y|+1)+\omega_{2}(|y|)\right)
$$

for a.e. $t \in J$ and each $(x, y) \in(0, \infty) \times \mathbb{R}_{0}$.

Also define $\hat{g} \in C^{0}(\mathbb{R})$ by

$$
\hat{g}(u)= \begin{cases}g(u) & \text { for } u \in[0, \infty), \\ -g(-u)+2 g(0) & \text { for } u \in(-\infty, 0) .\end{cases}
$$

If $g$ satisfies assumption $\left(H_{1}\right)$, then $\hat{g}$ is increasing on $\mathbb{R}$, which is the domain of the inverse function $\hat{g}^{-1}$ to $\hat{g}$.

Consider the family of regular differential equations

$$
x^{\prime \prime}(t)=\lambda f_{n}\left(t, \hat{g}^{-1}(x(t)), x^{\prime}(t)\right)+(1-\lambda) a
$$

depending on the parameters $\lambda \in[0,1]$ and $n \in \mathbb{N}$, where $a$ appears in $\left(H_{3}\right)$.

LEMMA 3.1. Let assumptions $\left(H_{1}\right)$ and $\left(H_{3}\right)$ be satisfied and let $x$ be a solution of $B V P(\mathrm{E})_{n}^{\lambda}$, (1.2). Then there exists a unique $\xi \in(0, T)$ such that

(a) $x(\xi)=0$ and $x(t)>0$ for $t \in[0, \xi) \cup(\xi, T]$, 
(b) $x^{\prime}$ is increasing on $J, x^{\prime}(\xi)=0$ and $\left|x^{\prime}(t)\right| \geq a|\xi-t|$ for $t \in J$,

(c) $x(t) \geq \frac{a}{2}(t-\xi)^{2}$ for $t \in J$.

Proof. By (3.1),

$$
x^{\prime \prime}(t) \geq a \text { for a.e. } t \in J
$$

From (3.3) it follows that $x^{\prime}$ is increasing on $J$ and then $x(0)=x(T)$ implies that $x^{\prime}$ vanishes at a unique point $\xi \in(0, T)$ and $x$ is decreasing on $[0, \xi]$ and increasing on $[\xi, T]$. Hence the condition $\min \{x(t): t \in J\}=0$ yields $x(\xi)=0$ and $x>0$ on $[0, \xi) \cup(\xi, T]$. The validity of the inequalities in (b) and (c) follows immediately by integration of (3.3) and using $x(\xi)=x^{\prime}(\xi)=0$.

REMARK 3.2. Lemma 3.1 shows that any solution $x$ of BVP $(\mathrm{E})_{n}^{\lambda},(1.2)$ with $\lambda \in$ $[0,1]$ and $n \in \mathbb{N}$ satisfies the inequality $x(t)>0$ for $t \in[0, \xi) \cup(\xi, T]$ where $\xi \in(0, T)$ is the unique zero of $x$. Hence $\hat{g}^{-1}(x(t))=g^{-1}(x(t))$ for $t \in J$.

LEMMA 3.3. Let assumptions $\left(H_{1}\right)$ and $\left(H_{3}\right)-\left(H_{5}\right)$ be satisfied. Let $x$ be a solution of $B V P(\mathrm{E})_{n}^{\lambda}$, (1.2). Then there exists a positive constant $P$ independent of $\lambda \in[0,1]$ and $n \in \mathbb{N}$ such that

$$
\|x\|=\sup _{t \in J}|x(t)|<P, \quad\left\|x^{\prime}\right\|<P .
$$

Proof. By Lemma 3.1, there exists a unique $\xi \in(0, T)$ such that $x(\xi)=x^{\prime}(\xi)=0$, $x(t)>0$ on $[0, \xi) \cup(\xi, T]$ and $x^{\prime}$ is increasing on $J$. Hence

$$
\|x\|=x(0)(=x(T)), \quad\left\|x^{\prime}\right\|=\max \left\{\left|x^{\prime}(0)\right|, x^{\prime}(T)\right\} .
$$

In addition (see (3.2) and Remark 3.2)

$$
x^{\prime \prime}(t) \leq\left[h_{1}\left(g^{-1}(x(t))+1\right)+h_{2}\left(g^{-1}(x(t))\right)\right]\left[\omega_{1}\left(\left|x^{\prime}(t)\right|+1\right)+\omega_{2}\left(\left|x^{\prime}(t)\right|\right)\right]
$$

for a.e. $t \in J$. Integrating the inequality (for a.e. $t \in[0, \xi)$ )

$$
\frac{x^{\prime \prime}(t) x^{\prime}(t)}{\omega_{1}\left(-x^{\prime}(t)+1\right)+\omega_{2}\left(-x^{\prime}(t)\right)} \geq\left[h_{1}\left(g^{-1}(x(t))+1\right)+h_{2}\left(g^{-1}(x(t))\right)\right] x^{\prime}(t)
$$

from $t \in[0, \xi)$ to $\xi$, we get

$$
\int_{0}^{-x^{\prime}(t)} \frac{s}{\omega_{1}(s+1)+\omega_{2}(s)} d s \leq \int_{0}^{x(t)}\left[h_{1}\left(g^{-1}(s)+1\right)+h_{2}\left(g^{-1}(s)\right)\right] d s .
$$

Hence $K\left(-x^{\prime}(t)\right) \leq H_{1}(x(t))$, where $K$ and $H_{1}$ are defined by (1.5) and (1.6), respectively. Then

$$
-x^{\prime}(t) \leq K^{-1}\left(H_{1}(x(t))\right) \text { for } t \in[0, \xi]
$$

and integrating

$$
-\frac{x^{\prime}(t)}{K^{-1}\left(H_{1}(x(t))\right)} \leq 1 \quad(\text { where } 0 \leq t<\xi)
$$


over $[0, \xi]$, we have

$$
\int_{0}^{x(0)} \frac{1}{K^{-1}\left(H_{1}(s)\right)} d s \leq \xi
$$

Arguing as above on the inequality (for a.e. $t \in[\xi, T]$ )

$$
\frac{x^{\prime \prime}(t) x^{\prime}(t)}{\omega_{1}\left(x^{\prime}(t)+1\right)+\omega_{2}\left(x^{\prime}(t)\right)} \leq\left[h_{1}\left(g^{-1}(x(t))+1\right)+h_{2}\left(g^{-1}(x(t))\right)\right] x^{\prime}(t)
$$

now on the interval $[\xi, T]$, we get

$$
x^{\prime}(t) \leq K^{-1}\left(H_{1}(x(t))\right) \quad \text { for } t \in[\xi, T]
$$

and

$$
\int_{0}^{x(T)} \frac{1}{K^{-1}\left(H_{1}(s)\right)} d s \leq T-\xi .
$$

Then (3.5), (3.8) and (3.10) imply

$$
\int_{0}^{\|x\|} \frac{1}{K^{-1}\left(H_{1}(s)\right)} d s \leq \frac{T}{2} .
$$

By $\left(H_{5}\right)$, there is a positive constant $V$ such that

$$
\int_{0}^{u} \frac{1}{K^{-1}\left(H_{1}(s)\right)} d s>\frac{T}{2}
$$

for all $u \geq V$. Hence (3.11) yields $\|x\|<V$. Letting $t=0$ in (3.7), $t=T$ in (3.9) and using the last inequality, we get $-x^{\prime}(0)<K^{-1}\left(H_{1}(V)\right)$ and $x^{\prime}(T)<K^{-1}\left(H_{1}(V)\right)$. Then (see (3.5)) $\left\|x^{\prime}\right\|<K^{-1}\left(H_{1}(V)\right)$ and so (3.4) is true with $P=\max \left\{V, K^{-1}\left(H_{1}(V)\right)\right\}$.

LemmA 3.4. Let assumptions $\left(H_{1}\right)$ and $\left(H_{3}\right)-\left(H_{5}\right)$ be satisfied. Then BVP $(\mathrm{E})_{n}^{1}$, (1.2) has a solution $x$ for each $n \in \mathbb{N}$ and (3.4) is true with a positive constant $P$ given by Lemma 3.3 .

Proof. Fix $n \in \mathbb{N}$. Let

$$
\begin{gathered}
\Omega=\left\{(x, A):(x, A) \in C^{1}(J) \times \mathbb{R},\|x\|<\max \left\{P, \frac{a T^{2}}{4}\right\},\right. \\
\left.\left\|x^{\prime}\right\|<\max \left\{P, \frac{a T}{2}\right\},|A|<\max \left\{P, \frac{a T^{2}}{8}\right\}\right\}
\end{gathered}
$$

and the operator $\mathcal{S}: \bar{\Omega} \rightarrow C^{1}(J) \times \mathbb{R}$ be defined by the formula

$$
\mathcal{S}(x, A)=\left(A+\int_{0}^{T} S(t, s) f_{n}\left(s, \hat{g}^{-1}(x(s)), x^{\prime}(s)\right) d s, A+\min \{x(t): t \in J\}\right),
$$

where

$$
S(t, s)= \begin{cases}s\left(\frac{t}{T}-1\right) & \text { for } 0 \leq s \leq t \leq T \\ t\left(\frac{s}{T}-1\right) & \text { for } 0 \leq t<s \leq T\end{cases}
$$


We see that $S \in C^{0}(J \times J)$ and

$$
S(t, s)<0 \quad \text { for }(t, s) \in(0, T) \times(0, T) .
$$

Assume that $\left(x_{0}, A_{0}\right) \in \bar{\Omega}$ is a fixed point of $\mathcal{S}$; that is $\mathcal{S}\left(x_{0}, A_{0}\right)=\left(x_{0}, A_{0}\right)$. Then

$$
\begin{gathered}
x_{0}(t)=A_{0}+\int_{0}^{T} S(t, s) f_{n}\left(s, \hat{g}^{-1}\left(x_{0}(s)\right), x_{0}^{\prime}(s)\right) d s, \quad t \in J, \\
\min \left\{x_{0}(t): t \in J\right\}=0 .
\end{gathered}
$$

From (3.14) we deduce that $x_{0}(0)=x_{0}(T)\left(=A_{0}\right), x_{0} \in A C^{1}(J)$ and $x_{0}^{\prime \prime}(t)=$ $f_{n}\left(t, \hat{g}^{-1}\left(x_{0}(t)\right), x_{0}^{\prime}(t)\right)$ for a.e. $t \in J$. Hence $x_{0}$ is a solution of $\mathrm{BVP}(\mathrm{E})_{n}^{1},(1.2)$. Therefore, to prove the existence of a solution of $\mathrm{BVP}(\mathrm{E})_{n}^{1},(1.2)$ it is sufficient to verify that

$$
D(\mathcal{I}-\mathcal{S}, \Omega, 0) \neq 0
$$

where " $D$ " stands for the Leray-Schauder degree and $\mathcal{I}$ is the identity operator on $C^{1}(J) \times \mathbb{R}$. The validity of (3.16) will be proved by the homotopy property. We first define the operator $\mathcal{L}: \bar{\Omega} \times[0,1] \rightarrow C^{1}(J) \times \mathbb{R}$ by

$$
\mathcal{L}(x, A, \lambda)=\left(A+\frac{a}{2} t(t-T), A+(1-\lambda) x\left(\frac{T}{2}\right)+\lambda \min \{x(t): t \in J\}\right) .
$$

Then $\mathcal{L}$ is a continuous operator and also $\mathcal{L}(\bar{\Omega} \times[0,1])$ is relatively compact in $C^{1}(J) \times \mathbb{R}$. Set $\mathcal{V}=\mathcal{I}-\mathcal{L}(\cdot, \cdot, 0)$. Then $\mathcal{V}(x, A)=(x(t)-A-\operatorname{at}(t-T) / 2,-x(T / 2))$ for $(x, A) \in \bar{\Omega}$. We claim that $\mathcal{V}(-x,-A) \neq \nu \mathcal{V}(x, A)$, for all $(x, A) \in \partial \Omega$ and $v \in$ $[1, \infty)$, so that

$$
D(\mathcal{I}-\mathcal{L}(\cdot, \cdot, 0), \Omega, 0) \neq 0,
$$

by Theorem 8.3 in [5]. If not, there exist $\left(x_{*}, A_{*}\right) \in \partial \Omega$ and $\nu_{*} \in[1, \infty)$ such that $\mathcal{V}\left(-x_{*},-A_{*}\right)=v_{*} \mathcal{V}\left(x_{*}, A_{*}\right)$, we then have

$$
\begin{gathered}
-x_{*}(t)+A_{*}-\frac{a}{2} t(t-T)=v_{*}\left(x_{*}(t)-A_{*}-\frac{a}{2} t(t-T)\right), \quad t \in J, \\
x_{*}\left(\frac{T}{2}\right)=-v_{*} x_{*}\left(\frac{T}{2}\right) .
\end{gathered}
$$

From (3.20) we obtain that $x_{*}(T / 2)=0$ and then (3.19) with $t=T / 2$ gives $A_{*}=$ $\frac{v_{*}-1}{v_{*}+1} \frac{a T^{2}}{8}$. Hence $0 \leq A_{*}<a T^{2} / 8$, and so (see (3.19))

$$
\left|x_{*}(t)\right|=\left|A_{*}+\frac{a\left(v_{*}-1\right)}{2\left(v_{*}+1\right)} t(t-T)\right|<\frac{a T^{2}}{4}, \quad\left|x_{*}^{\prime}(t)\right|=\left|\frac{a\left(v_{*}-1\right)}{2\left(v_{*}+1\right)}(2 t-T)\right|<\frac{a T}{2} .
$$

We have proved that $\left(x_{*}, A_{*}\right) \notin \partial \Omega$ and so $(3.18)$ is true. Assume now that $\mathcal{L}(\hat{x}, \hat{A}, \hat{\lambda})=$ $(\hat{x}, \hat{A})$, for some $(\hat{x}, \hat{A}) \in \bar{\Omega}$ and $\hat{\lambda} \in[0,1]$. Then

$$
\begin{array}{r}
\hat{x}(t)=\hat{A}+\frac{a}{2} t(t-T), \quad t \in J, \\
(1-\hat{\lambda}) \hat{x}\left(\frac{T}{2}\right)+\hat{\lambda} \min \{\hat{x}(t): t \in J\}=0 .
\end{array}
$$


From (3.21) we conclude that $\hat{x}$ is a solution of equation $(\mathrm{E})_{n}^{0}, \hat{x}(0)=\hat{x}(T)(=\hat{A})$ and $\min \{\hat{x}(t): t \in J\}=\hat{x}(T / 2)$. Then (3.22) gives $\min \{\hat{x}(t): t \in J\}=0$, and so $\hat{x}$ is a solution of BVP (E) ${ }_{n}^{0}$, (1.2). By Lemma 3.3, $\|\hat{x}\|<P,\left\|\hat{x}^{\prime}\right\|<P$ and then $|\hat{A}|=|\hat{x}(0)|<$ $P$. Hence $(\hat{x}, \hat{A}) \notin \partial \Omega$. Thus (3.18) and the homotopy property yield

$$
D(\mathcal{I}-\mathcal{L}(\cdot, \cdot, 1), \Omega, 0)=D(\mathcal{I}-\mathcal{L}(\cdot, \cdot, 0), \Omega, 0) \neq 0 .
$$

Finally, define $\mathcal{K}: \bar{\Omega} \times[0,1] \rightarrow C^{1}(J) \times \mathbb{R}$ by

$$
\begin{gathered}
\mathcal{K}(x, A, \lambda)=\left(A+\int_{0}^{T} S(t, s)\left(\lambda f_{n}\left(s, \hat{g}^{-1}(x(s)), x^{\prime}(s)\right)+(1-\lambda) a\right) d s,\right. \\
A+\min \{x(t): t \in J\}) .
\end{gathered}
$$

Then $\mathcal{K}(\cdot, \cdot, 0)=\mathcal{L}(\cdot, \cdot, 1)$ and $\mathcal{K}(\cdot, \cdot, 1)=\mathcal{S}$. If we verify that

(i) $\mathcal{K}$ is a compact operator and

(ii) $\mathcal{K}(x, A, \lambda) \neq(x, A)$ for $(x, A) \in \partial \Omega$ and $\lambda \in[0,1]$,

then (3.23) guarantees the validity of (3.16). Since $f_{n} \in \operatorname{Car}\left(J \times \mathbb{R}^{2}\right)$, standard arguments show that $\mathcal{K}$ is a compact operator. To verify (ii), assume that $\mathcal{K}\left(x_{*}, A_{*}, \lambda_{*}\right)=\left(x_{*}, A_{*}\right)$, for some $\left(x_{*}, A_{*}\right) \in \bar{\Omega}$ and $\lambda_{*} \in[0,1]$. Then $x_{*}$ is a solution of $\operatorname{BVP}(\mathrm{E})_{n}^{\lambda_{*}},(1.2)$ and $x_{*}(0)=A_{*}$. According to Lemma 3.3, $\left\|x_{*}\right\|<P,\left\|x_{*}^{\prime}\right\|<P$ and then $\left|A_{*}\right|=\left|x_{*}(0)\right|<P$. Therefore $\left(x_{*}, A_{*}\right) \notin \partial \Omega$ and $\mathcal{K}$ has property (ii).

LEMMA 3.5. Let assumptions $\left(H_{1}\right)$ and $\left(H_{3}\right)-\left(H_{5}\right)$ be satisfied and let $x_{n}$ be a solution of $B V P(\mathrm{E})_{n}^{1},(1.2)$. Then the sequence

$$
\left\{f_{n}\left(t, g^{-1}\left(x_{n}(t)\right), x_{n}^{\prime}(t)\right)\right\} \subset L_{1}(J)
$$

is uniformly absolutely continuous (UAC) on $J$; that is for each $\varepsilon>0$ there exists $\delta>0$ such that

$$
\int_{\mathcal{M}} f_{n}\left(t, g^{-1}\left(x_{n}(t)\right), x_{n}^{\prime}(t)\right) d s<\varepsilon \quad(n \in \mathbb{N}),
$$

whenever $\mathcal{M} \subset J$ is measurable and $\mu(\mathcal{M})<\delta$, where $\mu(\mathcal{M})$ denotes the Lebesgue measure of $\mathcal{M}$.

Proof. By Lemmas 3.1 and 3.3,

$$
x_{n}(t) \geq \frac{a}{2}\left(\xi_{n}-t\right)^{2}, \quad\left|x_{n}^{\prime}(t)\right| \geq a\left|\xi_{n}-t\right| \quad \text { for } t \in J \text { and } n \in \mathbb{N},
$$

where $\xi_{n} \in(0, T), x_{n}\left(\xi_{n}\right)=x_{n}^{\prime}\left(\xi_{n}\right)=0$ and

$$
\left\|x_{n}\right\|<P,\left\|x_{n}^{\prime}\right\|<P \text { for } n \in \mathbb{N},
$$

where $P$ is a positive constant. Then $g^{-1}\left(x_{n}(t)\right)<g^{-1}(P)$ for $t \in J, n \in \mathbb{N}$ and (see (3.1) and (3.2))

$$
\begin{gathered}
a \leq f_{n}\left(t, g^{-1}\left(x_{n}(t)\right), x_{n}^{\prime}(t)\right) \\
\leq\left[h_{1}\left(g^{-1}(P)+1\right)+h_{2}\left(g^{-1}\left(x_{n}(t)\right)\right)\right]\left[\omega_{1}(P+1)+\omega_{2}\left(\mid x_{n}^{\prime}(t)\right)\right],
\end{gathered}
$$


for a.e. $t \in J$ and for $n \in \mathbb{N}$. Now, from (3.27) and the inequalities

$$
\begin{gathered}
h_{2}\left(g^{-1}\left(x_{n}(t)\right)\right) \omega_{2}\left(\left|x_{n}^{\prime}(t)\right|\right) \geq h_{2}\left(g^{-1}(P)\right) \omega_{2}\left(\left|x_{n}^{\prime}(t)\right|\right), \\
h_{2}\left(g^{-1}\left(x_{n}(t)\right)\right) \omega_{2}\left(\left|x_{n}^{\prime}(t)\right|\right) \geq h_{2}\left(g^{-1}\left(x_{n}(t)\right)\right) \omega_{2}(P),
\end{gathered}
$$

we see that the sequence (3.24) is UAC on $J$ if $\left\{h_{2}\left(g^{-1}\left(x_{n}(t)\right)\right) \omega_{2}\left(\left|x_{n}^{\prime}(t)\right|\right)\right\}$ is. From the structure of the measurable set on $J$ we deduce that the sequence $\left\{h_{2}\left(g^{-1}\left(x_{n}(t)\right)\right) \omega_{2}\left(\left|x_{n}^{\prime}(t)\right|\right)\right\}$ is UAC on $J$ if for each $\varepsilon>0$ there exists $\delta>0$ such that for any at most countable set $\left\{\left(a_{j}, b_{j}\right)\right\}_{j \in \mathbb{J}}$ of mutually disjoint intervals $\left(a_{j}, b_{j}\right) \subset J$, $\sum_{j \in \mathbb{J}}\left(b_{j}-a_{j}\right)<\delta$, we have

$$
\sum_{j \in \mathbb{J}} \int_{a_{j}}^{b_{j}} h_{2}\left(g^{-1}\left(x_{n}(t)\right)\right) \omega_{2}\left(\left|x_{n}^{\prime}(t)\right|\right) d t<\varepsilon \quad(n \in \mathbb{N}) .
$$

Therefore, let $\left\{\left(a_{j}, b_{j}\right)\right\}_{j \in \mathbb{J}}$ be an at most countable set of mutually disjoint intervals $\left(a_{j}, b_{j}\right) \subset J$ and set

$$
\mathbb{J}_{n}^{1}=\left\{j: j \in \mathbb{J},\left(a_{j}, b_{j}\right) \subset\left(0, \xi_{n}\right)\right\}, \mathbb{J}_{n}^{2}=\left\{j: j \in \mathbb{J},\left(a_{j}, b_{j}\right) \subset\left(\xi_{n}, T\right)\right\} .
$$

Then for $i \in \mathbb{J}_{n}^{1}$ and $j \in \mathbb{J}_{n}^{2}$ we have (see (3.25))

$$
\begin{aligned}
\int_{a_{i}}^{b_{i}} h_{2}\left(g^{-1}\left(x_{n}(t)\right)\right) \omega_{2}\left(\left|x_{n}^{\prime}(t)\right|\right) d s & \leq \int_{a_{i}}^{b_{i}} h_{2}\left(g^{-1}\left(\frac{a}{2}\left(\xi_{n}-t\right)^{2}\right)\right) \omega_{2}\left(a\left(\xi_{n}-t\right)\right) d t \\
& =\frac{1}{a} \int_{a\left(\xi_{n}-b_{i}\right)}^{a\left(\xi_{n}-a_{i}\right)} h_{2}\left(g^{-1}\left(\frac{s^{2}}{2 a}\right)\right) \omega_{2}(s) d s, \\
\int_{a_{j}}^{b_{j}} h_{2}\left(g^{-1}\left(x_{n}(t)\right)\right) \omega_{2}\left(\left|x_{n}^{\prime}(t)\right|\right) d s & \leq \int_{a_{j}}^{b_{j}} h_{2}\left(g^{-1}\left(\frac{a}{2}\left(\xi_{n}-t\right)^{2}\right)\right) \omega_{2}\left(a\left(t-\xi_{n}\right)\right) d t \\
& =\frac{1}{a} \int_{a\left(a_{j}-\xi_{n}\right)}^{a\left(b_{j}-\xi_{n}\right)} h_{2}\left(g^{-1}\left(\frac{s^{2}}{2 a}\right)\right) \omega_{2}(s) d s .
\end{aligned}
$$

If $a_{j_{n}}<\xi_{n}<b_{j_{n}}$ for some $j_{n} \in \mathbb{J}$, then

$$
\begin{aligned}
\int_{a_{j_{n}}}^{b_{j n}} h_{2}\left(g^{-1}\left(x_{n}(t)\right)\right) \omega_{2}\left(\left|x_{n}^{\prime}(t)\right|\right) d t \leq & \int_{a_{j_{n}}}^{\xi_{n}} h_{2}\left(g^{-1}\left(\frac{a}{2}\left(\xi_{n}-t\right)^{2}\right)\right) \omega_{2}\left(a\left(\xi_{n}-t\right)\right) d t \\
& +\int_{\xi_{n}}^{b_{j n}} h_{2}\left(g^{-1}\left(\frac{a}{2}\left(\xi_{n}-t\right)^{2}\right)\right) \omega_{2}\left(a\left(t-\xi_{n}\right)\right) d t \\
= & \frac{1}{a}\left[\int_{0}^{a\left(\xi_{n}-a_{j_{n}}\right)} h_{2}\left(g^{-1}\left(\frac{s^{2}}{2 a}\right)\right) \omega_{2}(s) d s\right. \\
& \left.+\int_{0}^{a\left(b_{j_{n}}-\xi_{n}\right)} h_{2}\left(g^{-1}\left(\frac{s^{2}}{2 a}\right)\right) \omega_{2}(s) d s\right] .
\end{aligned}
$$


Set

$$
\mathcal{M}_{n}^{1}=\mathcal{E}_{n}^{1} \cup \bigcup_{i \in \mathbb{J}_{n}^{1}}\left(a\left(\xi_{n}-b_{i}\right), a\left(\xi_{n}-a_{i}\right)\right), \quad \mathcal{M}_{n}^{2}=\mathcal{E}_{n}^{2} \cup \bigcup_{j \in \mathbb{J}_{n}^{2}}\left(a\left(a_{j}-\xi_{n}\right), a\left(b_{j}-\xi_{n}\right)\right),
$$

where

$$
\begin{aligned}
& \mathcal{E}_{n}^{1}= \begin{cases}\emptyset & \text { if } \mathbb{J}=\mathbb{J}_{n}^{1} \cup \mathbb{J}_{n}^{2}, \\
\left(0, a\left(\xi_{n}-a_{j_{n}}\right)\right) & \text { if }\left\{j_{n}\right\}=\mathbb{J} \backslash\left(\mathbb{J}_{n}^{1} \cup \mathbb{J}_{n}^{2}\right),\end{cases} \\
& \mathcal{E}_{n}^{2}= \begin{cases}\varnothing & \text { if } \mathbb{J}=\mathbb{J}_{n}^{1} \cup \mathbb{J}_{n}^{2}, \\
\left(0, a\left(b_{j_{n}}-\xi_{n}\right)\right) & \text { if }\left\{j_{n}\right\}=\mathbb{J} \backslash\left(\mathbb{J}_{n}^{1} \cup \mathbb{J}_{n}^{2}\right) .\end{cases}
\end{aligned}
$$

Then

$$
\begin{aligned}
& \sum_{j \in \mathbb{J}} \int_{a_{j}}^{b_{j}} h_{2}\left(g^{-1}\left(x_{n}(t)\right)\right) \omega_{2}\left(\left|x_{n}^{\prime}(t)\right|\right) d t \\
& \quad \leq \int_{\mathcal{M}_{n}^{1}} h_{2}\left(g^{-1}\left(\frac{s^{2}}{2 a}\right)\right) \omega_{2}(s) d s+\int_{\mathcal{M}_{n}^{1}} h_{2}\left(g^{-1}\left(\frac{s^{2}}{2 a}\right)\right) \omega_{2}(s) d s .
\end{aligned}
$$

By $\left(H_{5}\right), h_{2}\left(g^{-1}\left(s^{2} /(2 a)\right)\right) \omega_{2}(s) \in L_{1}([0, a T])$ and, since $\mu\left(\mathcal{M}_{n}^{k}\right) \leq a \sum_{j \in \mathbb{J}}\left(b_{j}-a_{j}\right)$ for $n \in \mathbb{N}$ and $k=1$, 2, we see that $\left\{h_{2}\left(g^{-1}\left(x_{n}(t)\right)\right) \omega_{2}\left(\left|x_{n}^{\prime}(t)\right|\right)\right\}$ is UAC on $J$ which finishes the proof.

\section{Existence results and examples.}

THEOREM 4.1. Let assumptions $\left(H_{1}\right)$ and $\left(H_{3}\right)-\left(H_{5}\right)$ be satisfied. Then BVP (1.1), (1.2) has a solution.

Proof. By Lemma 2.1 (see also Remark 2.2), the solvability of BVP (1.1), (1.2) is equivalent to that of BVP (2.1), (1.2). Theorem 4.1 will be proved if BVP (2.1), (1.2) has a solution.

By Lemma 3.4, BVP $(\mathrm{E})_{n}^{1}$, (1.2) has a solution $x_{n}$ for each $n \in \mathbb{N}$. Also Lemmas 3.1 and 3.3 guarantee the validity of inequalities (3.25) and (3.26), where $P$ is a positive constant and $\xi_{n} \in(0, T), x_{n}\left(\xi_{n}\right)=x_{n}^{\prime}\left(\xi_{n}\right)=0$. In addition (see Lemma 3.5), $\left\{f_{n}\left(t, g^{-1}\left(x_{n}(t)\right), x_{n}^{\prime}(t)\right)\right\}$ is UAC on $J$ and therefore $\left\{x_{n}^{\prime}(t)\right\}$ is equicontinuous on $J$. Going if necessary to a subsequence, we can assume, by the Arzelà-Ascoli theorem and the compactness principle, that $\left\{x_{n}\right\}$ is convergent in $C^{1}(J)$ and $\left\{\xi_{n}\right\}$ in $\mathbb{R}$. Let $\lim _{n \rightarrow \infty} x_{n}=x$ and $\lim _{n \rightarrow \infty} \xi_{n}=\xi$. Then $x$ satisfies the boundary conditions (1.2) and $(\operatorname{see}(3.25)) x(t) \geq(a / 2)(\xi-t)^{2},\left|x^{\prime}(t)\right| \geq a|\xi-t|$ for $t \in J$. Thus $x(t)>0$ and $\left|x^{\prime}(t)\right|>0$ for $t \in J \backslash\{\xi\}$, and $f\left(t, g^{-1}(x(t)), x^{\prime}(t)\right)$ is defined almost everywhere. Also

$$
\lim _{n \rightarrow \infty} f_{n}\left(t, g^{-1}\left(x_{n}(t)\right), x_{n}^{\prime}(t)\right)=f\left(t, g^{-1}(x(t)), x^{\prime}(t)\right) \quad \text { for a.e. } t \in J .
$$

Now, by the Vitali's convergence theorem, $f\left(t, g^{-1}(x(t)), x^{\prime}(t)\right) \in L_{1}(J)$ and

$$
\lim _{n \rightarrow \infty} \int_{0}^{t} f_{n}\left(s, g^{-1}\left(x_{n}(s)\right), x_{n}^{\prime}(s)\right) d s=\int_{0}^{t} f\left(s, g^{-1}(x(s)), x^{\prime}(s)\right) d s \quad(t \in J) .
$$


Letting $n \rightarrow \infty$ in the equalities

$$
x_{n}^{\prime}(t)=x_{n}^{\prime}(0)+\int_{0}^{t} f_{n}\left(s, g^{-1}\left(x_{n}(s)\right), x_{n}^{\prime}(s)\right) d s \quad(t \in J, n \in \mathbb{N}),
$$

we get

$$
x^{\prime}(t)=x^{\prime}(0)+\int_{0}^{t} f\left(s, g^{-1}(x(s)), x^{\prime}(s)\right) d s \quad(t \in J) .
$$

Hence $x \in A C^{1}(J)$ and $x$ is a solution of BVP (2.1), (1.2).

Corollary 4.2. Let assumptions $\left(\mathrm{H}_{2}\right)-\left(\mathrm{H}_{4}\right)$ and $\left(\mathrm{H}_{6}\right)$ be satisfied. Then BVP (1.3), (1.2) has a solution.

Proof. Using the function $G$ defined in (1.4) we can write equation (1.3) in the form

$$
\left(G(x(t))^{\prime \prime}=f\left(t, x(t),\left(G(x(t))^{\prime}\right),\right.\right.
$$

which is equation (1.1) with $G$ instead of $g$. Since assumption $\left(H_{6}\right)$ is obtained from assumption $\left(H_{5}\right)$ with $G$ instead of $g$, we see that BVP (4.1), (1.2) has a solution $x$, by Theorem 4.1, such that $x \in C^{0}(J)$ and $G(x) \in A C^{1}(J)$. Set $y(t)=G(x(t))$ for $t \in J$. Then $y \in A C^{1}(J)$ and from $x(t)=G^{-1}(y(t))$ we see that $x \in C^{1}(J)$ by $\left(H_{2}\right)$, and so $g(x) x^{\prime}=(G(x))^{\prime} \in A C(J)$. Consequently, $x$ is a solution of BVP (1.3), (1.2).

EXAMPLE 4.3. Consider the differential equation

$$
\left(x^{p}\right)^{\prime \prime}=c_{0}\left(1+c_{1} x^{\alpha}+\frac{c_{2}}{x^{\beta}}\right)\left(1+c_{3}\left|\left(x^{p}\right)^{\prime}\right|^{\gamma}+\frac{c_{4}}{\left|\left(x^{p}\right)^{\prime}\right|^{\delta}}\right),
$$

where $p \in(0, \infty), c_{0}, c_{2}, c_{4} \in(0, \infty), c_{1}, c_{3} \in[0, \infty), \alpha, \beta, \gamma \in(0, \infty), \delta \in(0,1)$ and $2 \beta<p(1-\delta)$. Equation (4.2) is the special case of (1.1) with $g(u)=u^{p}$ satisfying $\left(H_{1}\right)$, and

$$
f(t, x, y)=c_{0}\left(1+c_{1} x^{\alpha}+\frac{c_{2}}{x^{\beta}}\right)\left(1+c_{3}|y|^{\gamma}+\frac{c_{4}}{|y|^{\delta}}\right) .
$$

We see that $\left(H_{3}\right)$ is true with $a=\min \left\{1 / 2, c_{0}\right\}$ and $\left(H_{4}\right)$ with

$$
h_{1}(u)=c_{0}\left(1+c_{1} u^{\alpha}\right), \quad h_{2}(u)=\frac{c_{0} c_{2}}{u^{\beta}}, \quad \omega_{1}(u)=1+c_{3} u^{\gamma}, \quad \omega_{2}(u)=\frac{c_{4}}{u^{\delta}} .
$$

We now verify $\left(H_{5}\right)$. Notice that

$$
\int_{0}^{1} h_{2}\left(g^{-1}\left(s^{2}\right)\right) \omega_{2}(s) d s=c_{0} c_{2} c_{4} \int_{0}^{1} s^{-\left(\delta+\frac{2 \beta}{p}\right)} d s=\frac{p c_{0} c_{2} c_{4}}{(1-\delta) p-2 \beta}<\infty
$$

and by a calculation we can show that there exist positive constants $A, B$ and $u_{0} \in$ $(0, \infty)$ such that for $u \geq u_{0}$ we have

$$
H_{1}(u)=\int_{0}^{u}\left[h_{1}\left(g^{-1}(s)+1\right)+h_{2}\left(g^{-1}(s)\right)\right] d s< \begin{cases}A u^{\frac{p+\alpha}{p}} & \text { if } c_{1}>0 \\ A u & \text { if } c_{1}=0\end{cases}
$$




$$
K(u)=\int_{0}^{u} \frac{s}{\omega_{1}(s+1)+\omega_{2}(s)} d s> \begin{cases}B u^{2-\gamma} & \text { if } c_{3}>0 \\ B u^{2} & \text { if } c_{3}=0 .\end{cases}
$$

Hence there exists $u_{1} \geq u_{0}$ such that for $u \geq u_{1}$ we have

$$
K^{-1}\left(H_{1}(u)\right)< \begin{cases}\sqrt{\frac{A}{B}} u^{\frac{p+\alpha}{2 p}} & \text { if } c_{1}>0, c_{3}=0, \\ \sqrt{\frac{A}{B} u} & \text { if } c_{1}=0, c_{3}=0, \\ \sqrt[2-\gamma]{\frac{A}{B}} u^{\frac{p+\alpha}{p^{(2-\gamma)}}} & \text { if } c_{1}>0, c_{3}>0, \\ \sqrt[2-\gamma]{\frac{A}{B}} u^{\frac{1}{2-\gamma}} & \text { if } c_{1}=0, c_{3}>0 .\end{cases}
$$

Finally, from the last inequalities we deduce that if one of the cases

(a) $\alpha<p$ if $c_{1}>0, c_{3}=0$,

(b) $c_{1}=c_{3}=0$

(c) $\alpha<p(1-\gamma)$ if $c_{1}>0, c_{3}>0$,

(d) $\gamma \in(0,1)$ if $c_{1}=0$ and $c_{3}>0$

occurs, we have

$$
\lim _{u \rightarrow \infty} \int_{0}^{u} \frac{1}{K^{-1}\left(H_{1}(s)\right)} d s=\infty .
$$

Applying Theorem 4.1, BVP (4.2), (1.2) has a solution if one of the cases (a)-(d) is satisfied.

EXAMPLE 4.4. Consider the differential equation

$$
\left(\frac{x^{\prime}(t)}{(\max \{1, x(t)\})^{p}}\right)^{\prime}=c_{0}(x(t))^{\alpha}+\frac{c_{1}}{(x(t))^{\beta}}+\frac{c_{2}}{\left|x^{\prime}(t)\right|^{\gamma}},
$$

where $p \in(0,1), \alpha, \beta, \gamma, c_{i}$ are positive constants $(i=0,1,2)$ and

$$
2 \beta+\gamma<1, \quad \alpha<1-p
$$

Equation (4.4) is the special case of (1.3) with $g(u)=1 /(\max \{1, u\})^{p}$ satisfying $\left(H_{2}\right)$ since

$$
G(u)=\int_{0}^{u} g(s) d s= \begin{cases}u & \text { for } u \in[0,1] \\ \frac{u^{1-p}-p}{1-p} & \text { for } u \in(1, \infty)\end{cases}
$$

and

$$
f(t, x, y)=c_{0} x^{\alpha}+\frac{c_{1}}{x^{\beta}}+\frac{c_{2}}{(\max \{1, x\})^{p \gamma}|y|^{\gamma}} .
$$

We can see that $\left(H_{3}\right)$ is satisfied with $a=\min \left\{1 / 2, c_{0}, c_{1}\right\}$ and $\left(H_{4}\right)$ with

$$
h_{1}(u)=c u^{\alpha}, \quad h_{2}(u)=c\left(\frac{1}{u^{\beta}}+\frac{1}{(\max \{1, u\})^{p \gamma}}\right), \quad \omega_{1}(u)=1, \quad \omega_{2}(u)=\frac{1}{u^{\gamma}},
$$


where $c=\max \left\{c_{0}, c_{1}, c_{2}\right\}$. We shall show that (4.5) guarantees the validity of $\left(H_{6}\right)$. Since

$$
G^{-1}(u)= \begin{cases}u & \text { for } u \in[0,1] \\ \sqrt[1-p]{(1-p) u+p} & \text { for } u \in(1, \infty)\end{cases}
$$

we have

$$
\int_{0}^{1} h_{2}\left(G^{-1}\left(s^{2}\right)\right) \omega_{2}(s) d s=c \int_{0}^{1}\left(\frac{1}{s^{2 \beta+\gamma}}+\frac{1}{s^{\gamma}}\right) d s<\infty .
$$

Further for $u \geq 1$,

$$
\begin{aligned}
H_{2}(u)= & \int_{0}^{u}\left[h_{1}\left(G^{-1}(s)+1\right)+h_{2}\left(G^{-1}(s)\right)\right] d s=c \int_{0}^{1}\left[(s+1)^{\alpha}+\frac{1}{s^{\beta}}+1\right] d s \\
& +c \int_{1}^{u}\left[(\sqrt[1-p]{(1-p) s+p}+1)^{\alpha}+\frac{1}{(\sqrt[1-p]{(1-p) s+p})^{\beta}}\right. \\
& \left.+\frac{1}{(\sqrt[1-p]{(1-p) s+p})^{p \gamma}}\right] d s
\end{aligned}
$$

and, for $u \geq 0$, we have

$$
K(u)=\int_{0}^{u} \frac{s}{\omega_{1}(s+1)+\omega_{2}(s)} d s=\int_{0}^{u} \frac{s^{1+\gamma}}{1+s^{\gamma}} d s .
$$

Thus there exist a positive constant $A$ and $u_{1} \in(1, \infty)$ such that

$$
H_{2}(u)<A u^{1+\frac{\alpha}{1-p}}, \quad K(u)>A u^{2} \quad \text { for } u \geq u_{1} .
$$

Now from (4.6) we deduce that

$$
K^{-1}\left(H_{2}(u)\right)<\sqrt{u^{1+\frac{\alpha}{1-p}}} \quad\left(u \geq u_{2}\right),
$$

where $u_{2}\left(\geq u_{1}\right)$ is a sufficiently large number. Since $\alpha<1-p$ by (4.5), we see that

$$
\lim _{u \rightarrow \infty} \int_{0}^{u} \frac{1}{K^{-1}\left(H_{2}(s)\right)} d s=\infty .
$$

We have verified that $\left(H_{6}\right)$ is true. Applying Theorem 4.1, BVP (4.4), (1.2) has a solution.

ACKNOWLEDGEMENTS. The authors are grateful to the referee for his suggestions on the first draft of this paper.

\section{REFERENCES}

1. R. P. Agarwal, D. O'Regan and S. Staněk, An upper and lower solution theory for the problem $\left(G^{\prime}(y)\right)^{\prime}+f(t, y)=0$ on finite and infinite intervals, (to appear).

2. R. P. Agarwal and S. Staněk, Existence of positive solutions to singular semi-positone boundary value problems, Nonlinear Anal. 51 (2002), 821-842.

3. R. G. Bartle, A modern theory of integration (AMS Providence, Rhode Island 2001). 
4. A. Cabada and R. L. Pouso, Existence theory for functional $p$-Laplacian equations with variable exponents, Nonlinear Anal. 52 (2003), 557-572.

5. K. Deimling, Nonlinear functional analysis (Springer-Verlag, 1985).

6. E. Hewitt and K. Stromberg, Real and abstract analysis (Springer-Verlag, 1965).

7. D. O'Regan, Existence theory for the equations $\left(G^{\prime}(y)\right)^{\prime}=q f\left(t, y, y^{\prime}\right)$ and $\left(G^{\prime}(y)-\right.$ $p H(y))^{\prime}=-p^{\prime} H(y)+q f(t, y)$, J. Math. Anal. Appl. 183 (1994), 435-470.

8. I. Rachůnková and S. Staněk, Connections between types of singularities in differential equations and smoothness of solutions for Dirichlet BVPs, Dynamics of Continuous, Discrete and Impulsive Systems, Series A: Math. Anal. 10 (2003), 209-222.

9. I. Rachůnková and S. Staněk, Sign-changing solutions of singular Dirichlet boundary value problems, Archives of Inequalities and Applications 1 (2003), 11-30.

10. S. Staněk, Positive solutions of singular Dirichlet and periodic boundary value problems, Computers and Mathematics with Applications 43 (2002), 681-692. 